\title{
Analysis of anterior trunk symmetry index (ATSI). Preliminary report
}

\author{
L Stolinski $1^{*}$, T Kotwicki ${ }^{2}$ D Czaprowski ${ }^{1,2,3}$, J Chowanska ${ }^{1,2}$ \\ From 9th International Conference on Conservative Management of Spinal Deformities - SOSORT 2012 \\ Annual Meeting \\ Milan, Italy. 10-12 May 2012
}

\section{Background}

Spinal deformities and postural disorders can be assessed by evaluation of trunk surface deformity. Usually, the back shape is assessed. However, the anterior trunk can also develop deformity, which is observed by the patient more easily than the back surface.

\section{Aim of the study}

To introduce a new parameter, Anterior Trunk Symmetry Index (ATSI), for anterior trunk deformity assessment.

\section{Methods}

Seventy primary school children, free of idiopathic scoliosis, both sexes, aged 6-7 years, mean $6.9 \pm 0.3$ years were examined with digital photography of their trunk, taken from the front in standardized conditions. The anatomical landmarks were: sternal notch, acromions, axilla folds, waist lines, and umbilicus. ATSI was defined as the sum of six indices: three frontal plane asymmetry indices (one for sternal notch, axilla folds and waist lines, respectively) and three height difference indices, (one for acromions, axilla folds, and waist lines, respectively). The software was developed for semi-automatic calculation of ATSI, after the anatomical points are indicated on a digital photo by the observer. The intra-observer error was calculated by the first author, by measuring four times the pictures of 20 children, in the interval of at least one day. The inter-observer error was calculated by one surgeon, and three experienced physiotherapists, by measuring the pictures of 20 children. The normal value limit was calculated as mean $\pm 3 \mathrm{SD}$.

\section{Results}

The assessment of the ATSI on digital photography took around 1 minute. The mean ATSI value for 70 children was $22.6 \pm 10.8$. The intra-observer error was 1.23 . The inter-observer error for the four observers was 3.08. The normal value limit was 32.3 .

\section{Conclusion}

This new surface parameter can be easily calculated on regular digital photographs of the anterior trunk. Both intra- and inter-observer errors are small, indicating possible reliability of ATSI for the assessment of anterior trunk asymmetry in children. Further studies are needed to assess the clinical usefulness of the ATSI parameter.

\section{Author details}

'Rehasport Clinic, Poznan, Poland. ${ }^{2}$ Spine Disorders Unit, Department of Pediatric Orthopedics and Traumatology, University of Medical Sciences, Poznan, Poland. ${ }^{3}$ The Faculty of Physiotherapy, Jozef Rusiecki University, Olsztyn, Poland.

Published: 3 June 2013

\section{References}

1. Suzuki N, I K, Ono T, Kohno K, Asher MA: Analysis of posterior trunk symmetry index (POTSI) in scoliosis, part 1. Stud Health Technol Inform 1999, 59:81-84.

2. Inami K, S N, Ono T, Yamashita Y, Kohno K, Morisue H: Analysis of posterior trunk symmetry index (POTSI) in scoliosis, part 2. Stud Health Technol Inform 1999, 59:85-88.

3. Minguez MF, Buendia M, Cibrian RM, Salvador R, Laguia M, Martin A, Gomar F: Quantifier variables of the back surface deformity obtained with a noninvasive structured light method: evaluation of their usefulness in idiopathic scoliosis diagnosis. Eur Spine J 2007, 16(1):73-82.

doi:10.1186/1748-7161-8-S1-O25

Cite this article as: Stolinski et al:: Analysis of anterior trunk symmetry index (ATSI). Preliminary report. Scoliosis 2013 8(Suppl 1):O25.

${ }^{1}$ Rehasport Clinic, Poznan, Poland

Full list of author information is available at the end of the article

C 2013 Stolinski et al; licensee BioMed Central Ltd. This is an Open Access article distributed under the terms of the Creative Commons 